\title{
STRUCTURALLY STABLE DIFFEOMORPHISMS ARE DENSE
}

\author{
BY MICHAEL SHUB ${ }^{1}$ \\ Communicated by Robert Seeley, February 15, 1972
}

Let $M$ be a $C^{\infty}$ compact manifold without boundary. Let $f \in \operatorname{Diff}^{r}(M)$, $1 \leqq r \leqq \infty$. $f$ is structurally stable in $\operatorname{Diff}^{r}(M)$ if there exists a neighborhood $U_{f}$ of $f$ in $\operatorname{Diff}^{r}(M)$ such that given $g \in U_{f}$ there exists a homeomorphism $h: M \rightarrow M$ such that $h f=g h$. The structurally stable diffeomorphisms are known not to be dense in $\operatorname{Diffr}^{r}(M)$, unless $M$ is the circle (see [4], [5], etc.). On the other hand I will prove below the mixed result that the structurally stable diffeomorphisms are always dense in $\operatorname{Diff}^{r}(M)$ with the $C^{0}$ topology. The main tool is the theorem of Smale [6], that every diffeomorphism is isotopic to a structurally stable diffeomorphism. Theorem 2 improves this theorem by producing an isotopy which is arbitrarily small in the $C^{0}$ topology. I expect to prove a corresponding theorem for vector fields with $\mathrm{M}$. W. Hirsch. It is a pleasure to acknowledge helpful conversations with $\mathrm{M}$. W. Hirsch and S. Smale. Let $m=\operatorname{dim} M$.

THEOREM 1 . Let $1 \leqq r \leqq \infty$. Then the structurally stable diffeomorphisms are dense in $\operatorname{Diff}^{r}(M)$ with the $C^{0}$ topology.

A sharper version of this theorem is

THEOREM 2. Let $1 \leqq r \leqq \infty$. Let $f \in \operatorname{Diff}^{r}(M)$. Then $f$ is $C$ isotopic to a structurally stable diffeomorphism $g$ by an isotopy which is arbitrarily small in the $C^{0}$ topology.

The following proposition is actually part of the proof of the main theorem of [6].

Proposition (SMale). Let $f \in \operatorname{Diff}^{r}(M)$. Let $M=H_{m} \supset H_{m-1} \supset \cdots$ $\supset H_{1} \supset H_{0}$ be a handle body decomposition of $M$ (corresponding to a "nice" Morse function). Suppose $f\left(H_{i}\right) \subset$ interior $H_{i}$ for all $i$. Then $f$ is $C^{r}$ isotopic to a structurally stable diffeomorphism.

The proof of this proposition in [6] is essentially complete, one need only take a little care in keeping track of the stable and unstable manifolds. Also, the $C^{0}$ size of the isotopy may be made small if the $i$-handles, $0 \leqq i \leqq m$, are small.

AMS 1970 subject classifications. Primary 58F10.

${ }^{1}$ Partially supported by NSF-GP 28375. 
Smale fixes a handle body decomposition of $M$ and isotopes any $f$ to satisfy the hypotheses of the proposition. The idea for Theorem 2 is to pick a fine handle body decomposition so that this first isotopy may also be made $C^{0}$ small.

ProOF OF THEOREM 2. Let $T$ be a triangulation of $M$ with small mesh (see [2]). Let $T_{i}, 0 \leqq i \leqq m$, be the $i$-skeleton of $T$. $f\left(T_{m-1}\right)$ misses a point in the interior of each $m$ simplex. By pushing away from this point we may isotope $f$ such that $f\left(T_{m-1}\right)$ is contained in an arbitrarily small neighborhood of $T_{m-1}$. By downward induction we may isotope $f$ to $g$ so that $g\left(T_{i}\right)$ is contained in an arbitrarily small neighborhood of $T_{i}$, $0 \leqq i \leqq m-1$. Now think of a small neighborhood $U_{0}$ of $T_{0}$ as the 0 -handles of a handle body decomposition of $M$, a small neighborhood $U_{1}$ of $T_{1}-U_{0}$ as the 1-handles, etc. Make $U_{i}$ so small that the $g\left(U_{i}\right)$ are already contained in prescribed small neighborhoods of the $T_{i}$. Now by upward induction on $i$ we may further isotope $g$ to preserve this handle body in the sense of the proposition. The $C^{0}$ size of the isotopy depends only on the mesh of $T$ and $m$.

Combining this theorem with [1] and [3], we see

THEOREM 3. Let $1 \leqq r<\infty$. Then there exists an open and dense set $U \subset \operatorname{Diff}^{r}(M)$ with the $C^{0}$ topology and a dense set of structurally stable diffeomorphisms $V \subset U$, such that the diffeomorphisms in $V$ are locally minimizing for the topological entropy of the diffeomorphisms in $U$.

This theorem may be of some interest to ecologists and others who sometimes try to achieve stability by maximizing the entropy.

\section{REFERENCES}

1. R. L. Adler, A. G. Konheim and M. H. McAndrew, Topological entropy, Trans. Amer. Math. Soc. 114 (1965), 309-319. MR 30 \#5291.

2. J. R. Munkres, Elementary differential topology, Ann. of Math. Studies, no. 54, Princeton Univ. Press, Princeton, N.J., 1963; rev. ed., 1966. MR 29 \# 623; 33 \# 6637.

3. Z. Nitecki, On semi-stability for diffeomorphisms, Invent. Math. 14 (1971), 83-123.

4. M. M. Peixoto, Structural stability on two-dimensional manifolds, Topology 1 (1962), 101-120. MR 26 \#426.

5. S. Smale, Structurally stable systems are not dense, Amer. J. Math. 88 (1966), 491-496. MR 33 \# 4911.

6. - Stability and isotopy in discrete dynamical systems, Proc. Internat. Sympos. on Dynamical Systems (Salvador, Brazil, 1971) (to appear).

Department of Mathematics, Brandeis University, Waltham, MassachusetTs 02154 